\title{
Histologic Findings in Mucosa and Muscularis Propria Biopsied During Peroral Endoscopic Myotomy in Patients With Achalasia
}

\author{
Xiuli Liu ${ }^{\mathrm{a}, \mathrm{d}}$, Enoch Kuo ${ }^{\mathrm{b}}$, Kai Wang ${ }^{\mathrm{b}}$, Yassen B. Perbtani ${ }^{\mathrm{c}}$, \\ Dennis Yang ${ }^{\mathrm{c}}$, Peter Draganov ${ }^{\mathrm{c}}$
}

\begin{abstract}
Background: Peroral endoscopic myotomy (POEM) has been increasingly used to treat achalasia. Previous studies have reported high frequency of muscular eosinophilic infiltration in achalasia. Esophageal mucosal changes in achalasia have only been studied in esophagectomy specimens. Cardia mucosal changes in achalasia have not been reported previously. We aimed to further characterize the esophageal, gastric cardia, and muscularis propria changes in achalasia.

Methods: This was a pilot study. Patients with clinically and radiographically confirmed achalasia who underwent POEM were enrolled in the study. Mucosal biopsies were taken $1 \mathrm{~cm}$ proximal and $1 \mathrm{~cm}$ distal to the gastroesophageal junction, and muscularis propria biopsies were taken from the mid esophagus. Tissues were submitted for histological evaluation.
\end{abstract}

Results: Eighteen patients (10 male and eight female, mean age: 60.7 (standard deviation (SD): 13) years) were enrolled in this pilot study. Nine patients had type II achalasia, two type III, one type I, five esophageal gastric outlet obstruction, and one unspecific type achalasia. The mean duration of symptoms prior to POEM was 79 (range 1 480) months. All patients had a dilated esophagus on examination, but no endoscopic evidence of Barrett's esophagus. Esophageal, gastric cardia, and muscular biopsies were performed in 17, 13, and 17 patients, respectively. Basal hyperplasia, spongiosis, ballooning, and parakeratosis were seen in $92.3 \%, 100 \%, 100 \%$, and $76.5 \%$ of cases, respectively. Intraepithelial lymphocytosis was seen in $70.5 \%$ of cases, and active esophagitis was seen in $23.5 \%$ of case. Six $(35.3 \%)$

Manuscript submitted July 28, 2021, accepted August 24, 2021

Published online August 31, 2021

aDepartment of Pathology and Immunology, Washington University School of Medicine, St. Louis, MO, USA

bDepartment of Pathology, Immunology, and Laboratory Medicine, College of Medicine, University of Florida, Gainesville, FL, USA

'Division of Gastroenterology, Department of Internal Medicine, College of Medicine, University of Florida, Gainesville, FL, USA

${ }^{\mathrm{d} C}$ Corresponding Author: Xiuli Liu, Department of Pathology and Immunology, Washington University School of Medicine, 660 S. Euclid Avenue, St. Louis, MO 63110,USA.Email: 1.xiuli@wustl.edu

doi: https://doi.org/10.14740/gr1454 cases had few intraepithelial eosinophils, but none of them had $>15$ eosinophils per high power field. Histologic findings in gastric cardia mucosa included carditis $(69.2 \%), H$. pylori gastritis $(7.6 \%)$, and reactive gastropathy $(15.4 \%)$. One case $(7.6 \%)$ showed low-grade dysplasia arising from intestinal metaplasia in the cardia. Absence of ganglion cells in the muscular biopsies was noted in $88.2 \%$ of cases, and the remaining two showed rare residual ganglion cells with ganglionitis in one case (5.8\%). Muscular atrophy and interstitial fibrosis were observed in $52.9 \%$ and $82.3 \%$ of the cases, respectively. Two cases $(11.7 \%)$ had eosinophilic inflammation in the muscularis propria and one of them was accompanied by lymphocytic inflammation.

Conclusions: Muscular biopsies in our study revealed loss of ganglion cells, supporting the view that achalasia is a primary esophageal disease with ganglion cell depletion. Squamous mucosa in achalasia showed changes mimicking reflux and lymphocytic esophagitis. Cardia mucosa in achalasia patients often were inflamed and uncommonly showed intestinal metaplasia and glandular dysplasia.

Keywords: Achalasia; Eosinophilic esophagitis; Ganglion cell; Lymphocytic esophagitis; Peroral endoscopic myotomy

\section{Introduction}

Achalasia is a rare disease characterized by aperistalsis of the esophageal body and failure of the lower esophageal sphincter to relax with attempted swallowing. Patients with achalasia typically present with dysphagia. Diagnosis is often made by timed barium esophagram, high-resolution manometry and endoscopy. Treatment options for symptomatic achalasia include botulinum toxin injection, pneumatic balloon dilation and endoscopy or surgical myotomy.

The etiology of achalasia remains largely unknown. In some patients with achalasia, myenteric inflammation has been associated with eosinophilic/lymphocytic infiltration of the esophageal muscle in resected esophagectomy or myotomy specimens [1-3]. Other common findings include severe fibrosis and obvious myopathic changes of the smooth muscle cells [3]. However, the significance of these findings has not been corroborated in large patient population studies, particularly in the early stage of disease or in patients treated endoscopi- 
cally before the development of end stage achalasia. It is well known that both eosinophilic and lymphocytic esophagitis, characterized by infiltration of eosinophils and lymphocytes in the mucosa, can manifest clinically with dysphagia. Whether eosinophilic/lymphocytic mural infiltration of the muscle layer of the esophagus is routinely present and plays a role in achalasia remains to be determined $[4,5]$. Recent literature suggests an association of achalasia with eosinophilic esophagitis [4].

Peroral endoscopic myotomy (POEM) has become a standard endoscopic therapy of achalasia [6]. During the POEM procedure, biopsy of the mucosa and the muscle layer of the esophagus can be easily obtained. We hypothesize that eosinophilic/lymphocytic esophagitis and achalasia potentially represent spectrums of the same condition. The aim of the study was to examine the eosinophilic and lymphocytic infiltration in esophageal mucosa and muscle on biopsy taken simultaneously during POEM procedure done for therapy of clinically and radiographically confirmed achalasia as part of routine patient care.

\section{Materials and Methods}

All adult patients (age 18 years or older) who were scheduled to undergo POEM at the University of Florida as part of their routine care were eligible for the study. The diagnosis of achalasia was attained based on high-resolution manometry findings according to the Chicago Classification of esophageal motility disorders along with endoscopic and radiographic findings. Exclusion criteria included: 1) any contraindication to perform endoscopy, 2) participation in another research protocol that could interfere or influence the outcomes measures of the present study, and 3) if the subject was unable/unwilling to give informed consent. The study was approved by the Institutional Research Board (IRB) at University of Florida. This study was conducted in compliance with the ethical standards of the responsible institution on human subjects as well as with the Helsinki Declaration.

\section{Biopsy and tissue handling protocol}

Biopsies of the esophageal mucosa ( $1 \mathrm{~cm}$ proximal to the gastroesophageal junction (GEJ)), gastric cardia mucosal biopsy ( $1 \mathrm{~cm}$ distal to the GEJ) were performed during the endoscopy for POEM. Biopsies of the muscularis propria at the mid esophagus level were performed after completion of the esophageal myotomy during POEM and prior to closure of the mucostomy.

The tissue specimens were fixed in formalin and embedded in paraffin and two $5-\mu \mathrm{m}$ sections were stained with hematoxylin and eosin (H\&E). The mucosal biopsies were analyzed for basal hyperplasia, elongation of papillae, spongiosis, ballooning, parakeratosis, squamous dysplasia, inflammation (peripapillary intraepithelial lymphocytes, interpapillary intraepithelial lymphocytes, neutrophil, eosinophil (peak and average), subepithelial fibrosis, subepithelial inflamma- tion, presence or absence of columnar epithelium, presence or absence of intestinal metaplasia, presence or absence of glandular dysplasia. The muscular biopsies were evaluated for smooth muscle atrophy, hypertrophy, anisocytosis, calcification, vacuolization, interstitial fibrosis, inflammation (eosinophil and lymphocyte), ganglionitis, neuritis, peri-vascular inflammation or intra-vascular inflammatory cell margination, presence or absence of ganglion cells. The histologic review was conducted using previously published methods with some modifications [2, 7-9]. Intraepithelial lymphocytosis was defined as more than 20 intraepithelial lymphocytes (IELs) per high power field (HPF) [10]. Definitions of histologic features evaluated in this study are listed in Tables 1 and 2. Data are presented as range (minimum - maximum) or mean \pm standard deviation (SD). Continuous data were compared with Student's $t$-test with significance setting at $\mathrm{P}<0.05$.

\section{Results}

\section{Demographics of study population}

Eighteen patients with achalasia were prospectively enrolled from June 2019 to June 2020 after providing informed consent in this pilot study. Clinical features and demographics of enrolled patients are summarized in Table 3. Eighteen patients (10 male and eight female, mean age: 60.7 (SD: 13; range 34 - 81) years) with achalasia were prospectively enrolled in this study. All patients had high-resolution manometry. Nine patients had type II achalasia, two type III, one type I, five esophageal gastric outlet obstruction, and one unspecific type achalasia. The mean duration of achalasia was 79 (SD: 114; range: 1 - 480) months. Two (11.0\%) patients had prior myotomy. Eleven (of 18, 61.1\%) patients were on proton pump inhibitors (PPIs) (omeprazole $40 \mathrm{mg}$ daily in six, $40 \mathrm{mg}$ bid in three, lansoprazole $15 \mathrm{mg}$ daily in one and PPI unspecified in one). Baseline timed barium esophagram was performed in 17 out of the 18 patients and revealed dilated esophagus in six patients, dilated esophagus with delayed passage of barium in seven, and normal examination in four. All patients had a dilated esophagus on endoscopic examination, two patients had esophagitis and one with diverticulum, but none of them showed salmon-colored mucosa endoscopically. POEM was successfully completed in all of the patients without any complications.

\section{Histologic findings in esophageal mucosal biopsies}

Esophageal biopsies at the mid esophagus level were performed in 17 patients. The number of esophageal biopsy fragments ranged from 1 to 5 (median 3). The esophageal biopsy showed similar changes as summarized in Table 4. Basal hyperplasia, spongiosis, ballooning, and parakeratosis (Fig. 1a) were seen in $92.3 \%, 100 \%, 100 \%$, and $76.5 \%$ of cases, respectively. Mean peripapillary IELs and intrapapillary IELs were 37 (SD 29) and 22 (SD 21) per HPF, respectively. Twelve $(70.5 \%)$ cases had intraepithelial lymphocytosis (defined as $>$ 
Table 1. Definitions of Histologic Features Evaluated in the Mucosal Biopsies

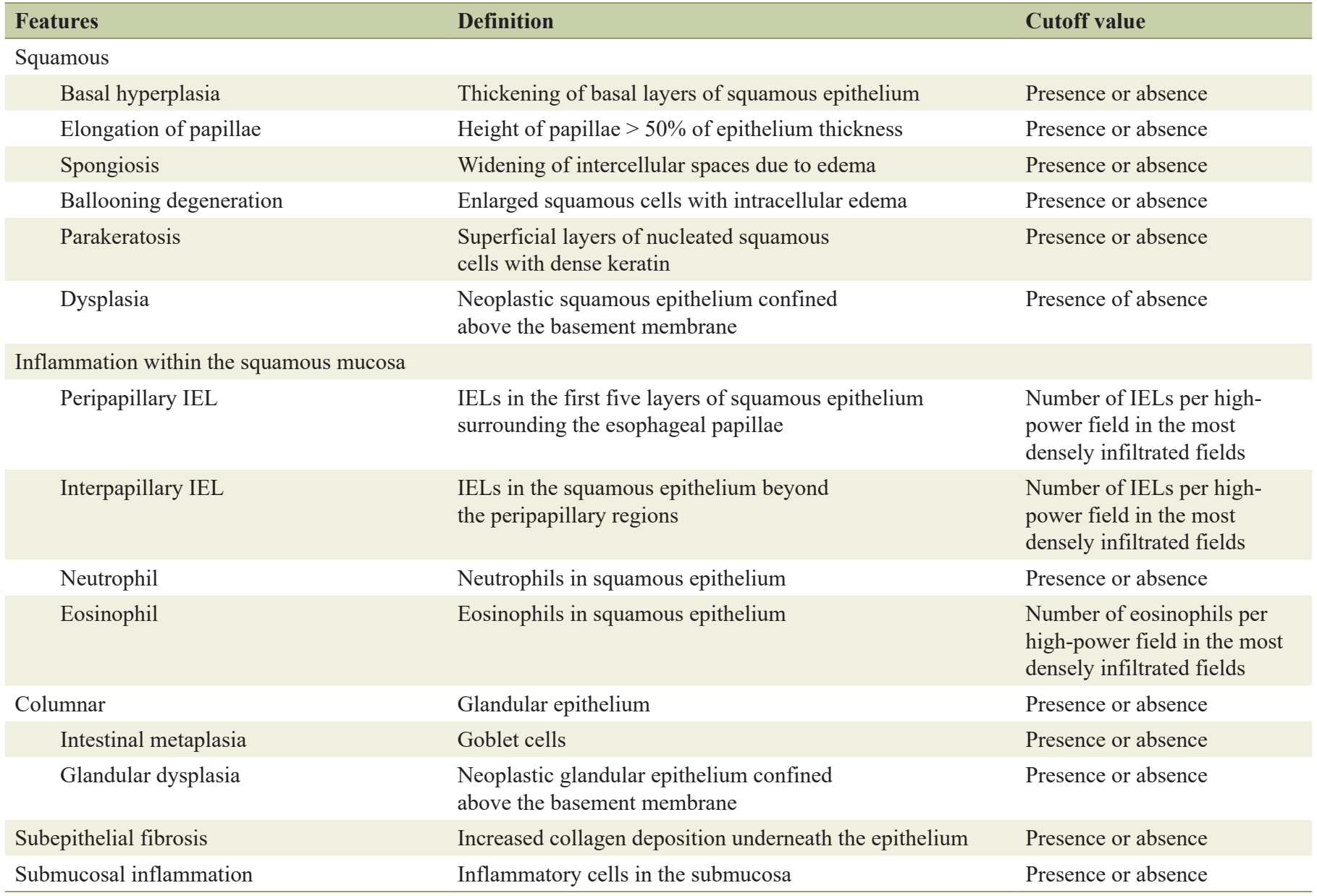

IEL: intraepithelial lymphocytosis; IELs: intraepithelial lymphocytes.

Table 2. Definitions of Histologic Features Evaluated in Muscular Biopsies

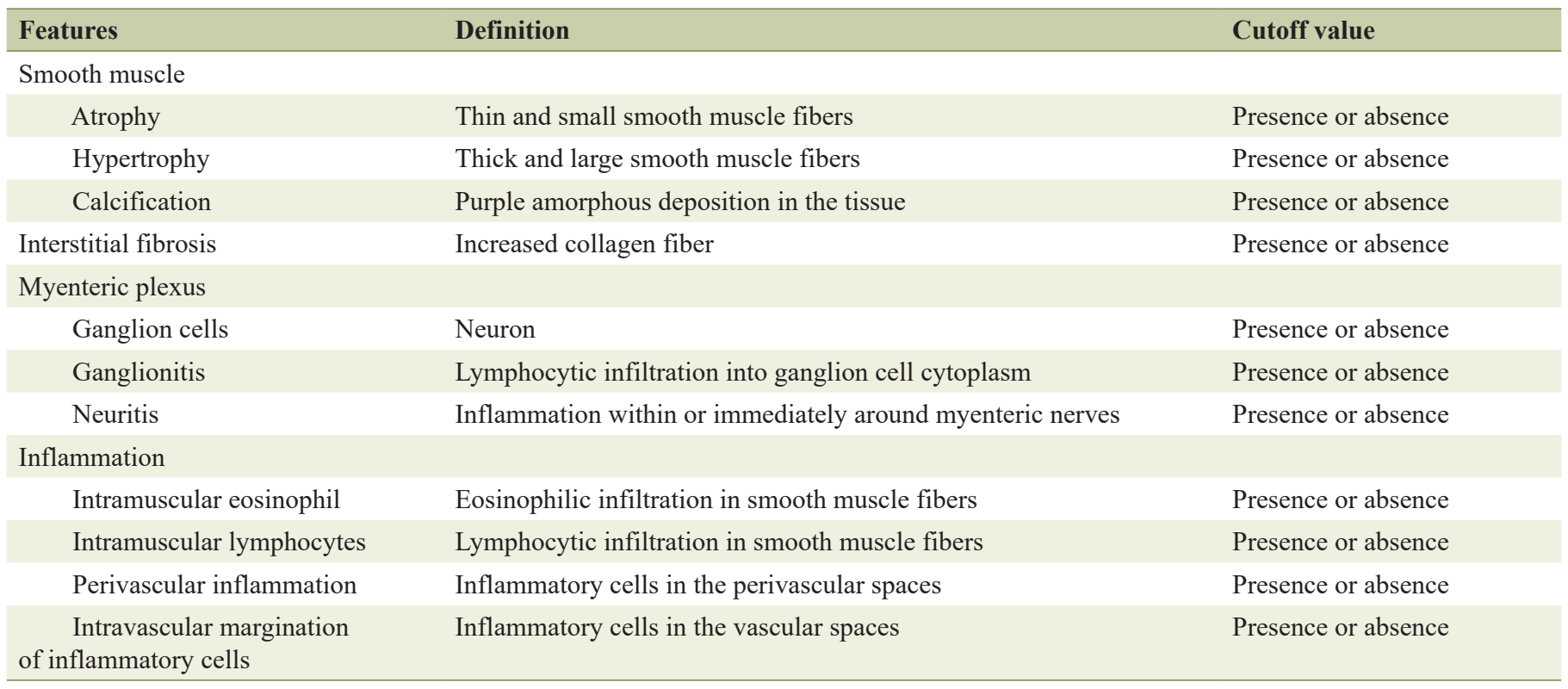


Table 3. Clinical Characteristics of Achalasia Patients

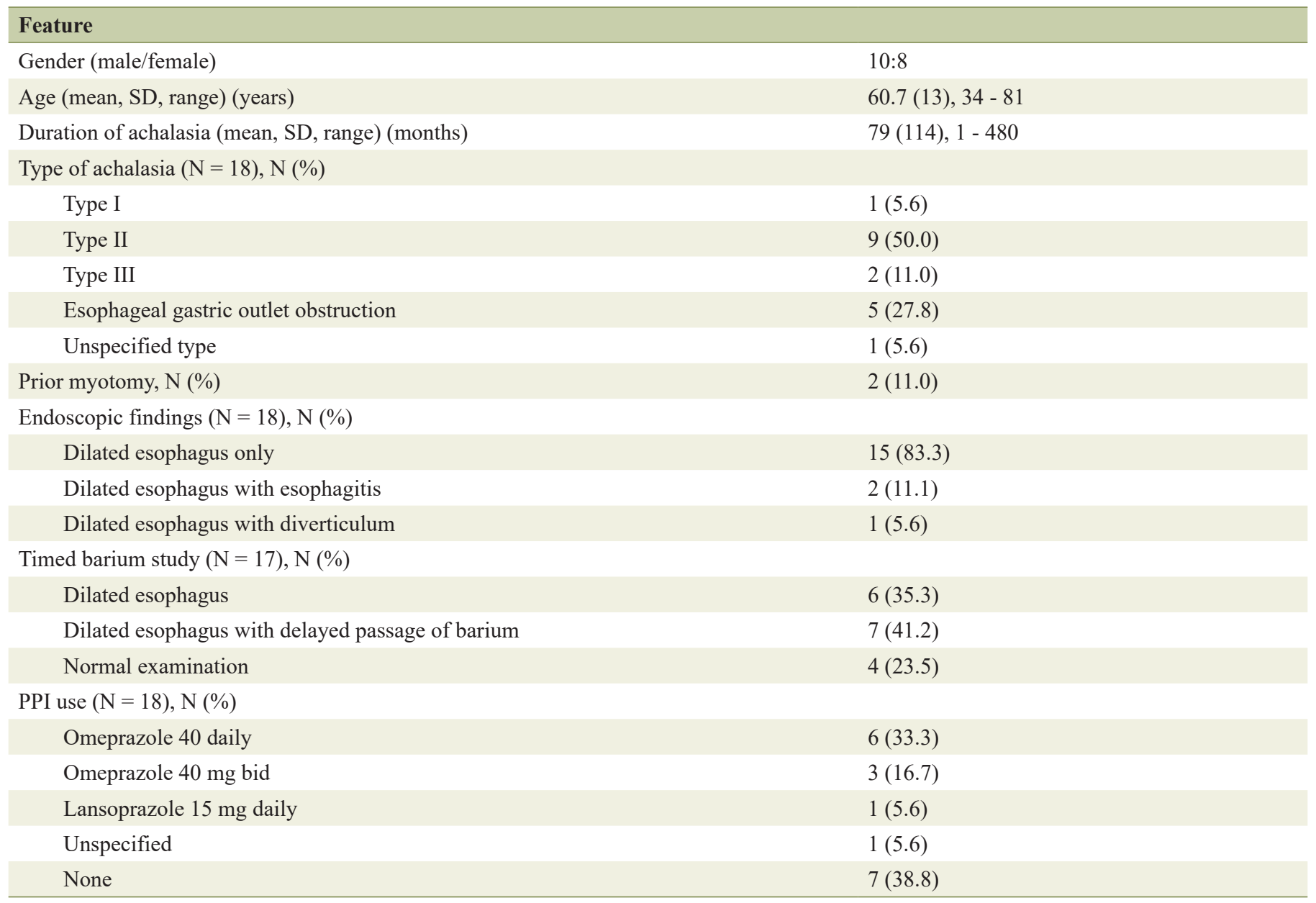

SD: standard deviation; PPI: proton pump inhibitor.

20 IELs per HPF) including eight cases with peripapillary and interpapillary lymphocytosis, two peripapillary intraepithelial lymphocytosis and two interpapillary lymphocytosis only. Peripapillary lymphocytosis was not associated with the use of PPI $(37 \pm 34$ IELs/HPF in patients taking PPIs vs. $33 \pm 321$ IELs/HPF in patients not taking PPIs, $\mathrm{P}=0.77$ ). Interpapillary lymphocytosis was not associated with the use of PPI either $(27 \pm 25 \mathrm{IELs} / \mathrm{HPF}$ in patients taking PPIs vs. $14 \pm 110 \mathrm{IELs} /$ $\mathrm{HPF}$ in patients not taking PPIs, $\mathrm{P}=0.26)$. Active esophagitis was seen in $23.5 \%$ of case. Six $(35.3 \%)$ cases had few intraepithelial eosinophils (Fig. 1b), but none of them had $>15$ eosinophils per HPF. Subepithelial inflammation and fibrosis were seen in $33.0 \%$ and $41.6 \%$ of cases, respectively. No squamous dysplasia was noted in any cases.

\section{Histologic findings in gastric cardia biopsies}

Gastric cardia biopsies were performed in 13 patients. The number of gastric cardia mucosal biopsy fragment ranged from 1 to 2 (median: 1). Histologic findings in gastric cardia mucosa included carditis (69.2\%) (Fig. 2a), H. pylori gastritis
(7.6\%), and reactive gastropathy (15.4\%) (Table 4). One case (7.6\%) had low-grade dysplasia (Fig. 2b) arising from intestinal metaplasia in the cardia which showed moderate chronic inactive carditis. No H. pylori organisms were seen on H\&Estained section in this case, but special stain or immunostain was not performed for $H$. pylori.

\section{Histologic findings in muscular biopsies}

Muscular biopsies were performed in 17 patients. The number of muscular biopsy fragments ranged from 1 to 4 (median: 2). The muscular biopsies showed similar changes in the study population and the results are summarized in Table 4. Absence of ganglion cells in the muscular biopsies was noted in $88.2 \%$ of cases, and the remaining two showed rare residual ganglion cells with ganglionitis and neuritis in one case (5.8\%). Muscular atrophy and interstitial fibrosis (Fig. 3a) were observed in $52.9 \%$ and $82.3 \%$ of cases, respectively. Two cases $(11.7 \%)$ had eosinophilic inflammation in the muscularis propria (Fig. $3 \mathrm{~b})$ and one of them was accompanied by lymphocytic inflammation. In these two cases, up to 25 and 50 eosinophils per 
Table 4. Esophageal, Gastric Cardia, and Muscular Biopsy Findings in Patients With Achalasia Undergoing Peroral Endoscopic Myotomy

\begin{tabular}{|c|c|c|}
\hline Mucosal biopsy findings & Feature & $\mathbf{N}(\%)$ \\
\hline \multirow[t]{4}{*}{ Esophageal squamous mucosa $(\mathrm{N}=17)$} & Basal hyperplasia & $16(92.3)$ \\
\hline & Spongiosis & $17(100)$ \\
\hline & Parakeratosis & $13(76.5)$ \\
\hline & Intraepithelial lymphocytosis & $12(70.5)$ \\
\hline \multirow[t]{4}{*}{ Gastric cardia mucosa $(\mathrm{N}=13)$} & Carditis & $9(69.2)$ \\
\hline & H. pylori gastritis & $1(7.6)$ \\
\hline & Reactive changes & $2(15.4)$ \\
\hline & Intestinal metaplasia & $1(7.6)$ \\
\hline \multirow{3}{*}{ Muscular biopsy findings $(\mathrm{N}=17)$} & Muscular atrophy & $9(52.9)$ \\
\hline & Interstitial fibrosis & $14(82.3)$ \\
\hline & Eosinophilic inflammation & $2(11.7)$ \\
\hline
\end{tabular}

HPF were noted in the muscularis propria. One patient had esophageal gastric outlet obstruction for 1 year and the other patient had type III achalasia for 40 years. Five (29.4\%) cases showed focal and mild perivascular inflammatory infiltrate or intravascular inflammatory cell margination but without evidence of vasculitis. None of the muscular biopsies showed any calcification.

\section{Discussion}

Achalasia is a rare disease defined by aperistalsis of the esophageal body and failure of the lower esophageal sphincter to relax with attempted swallowing. Patients with achalasia typically present with dysphagia which may also be accompanied by other symptoms. Although advances in the diagnosis and treatment of achalasia have been made over the last decade, the etiology of achalasia remains elusive. This is partly due to the lack of knowledge on the histologic changes in early-stage achalasia. Histologic examination of esophagectomy and open Heller myotomy specimens have shown myenteric inflammation, ganglion cell loss, and eosinophilic/lymphocytic infiltration of the esophageal muscle in achalasia [1,3]. Similar changes have been reported in deep muscle strips obtained during open Heller myotomy [2]. Other common findings include severe fibrosis and obvious myopathic changes of the smooth muscle cells [3]. However, the significance of these findings has not been corroborated in large patient population studies, particularly in early stage of disease or in patients treated endoscopically before the development of end-stage achalasia. This pilot study was carried out to examine myenteric plexus and muscular changes in achalasia patients undergoing POEM to obtain a snapshot view of the histologic changes at a stage earlier than those in esophagectomy and/or open Heller myotomy specimens as reported in previous studies. Notably, recent literature has suggested an association of achalasia with eosinophilic esophagitis [4]. As such, we also hypothesized that eosinophilic/lymphocytic infiltration of the esophageal wall layers could play a role in different disease processes, with eosinophilic/lymphocytic esophagitis and achalasia potentially representing spectrums of the same condition.

Muscular biopsies in our study revealed loss of ganglion cells in most cases, which is in line with previous reports in esophagectomy or myotomy specimens [1-3]. Interestingly, in our study, ganglionitis and neuritis was only noted in one case, albeit few ganglion cells were still present. This is in contrast to the high frequency of neuritis reported in other studies on esophagectomy and myotomy specimens $[1,2]$. We speculate that the discrepancy may have been due to the stage of achalasia, type of specimens (resection vs. small biopsy), and sample level (distal vs. mid esophagus). The mean duration of achalasia in our patient population was 79 months, longer than the 14-month duration of disease in two studies showing more prominent inflammation in myotomy specimens $[2,11]$. Sampling level (distal vs. mid esophagus) may not be the main reason as loss of ganglion cells and inflammation in myenteric plexus have been noted in the mid and distal esophagus in esophagectomy specimens [1]. However, the small size of muscular biopsy taking during POEM in the current study may have missed the area which was more inflamed. One recent study on esophageal nearly full-thickness muscle sample reported loss of Schwann cell loss which was more profound in achalasia type I and in the mid 

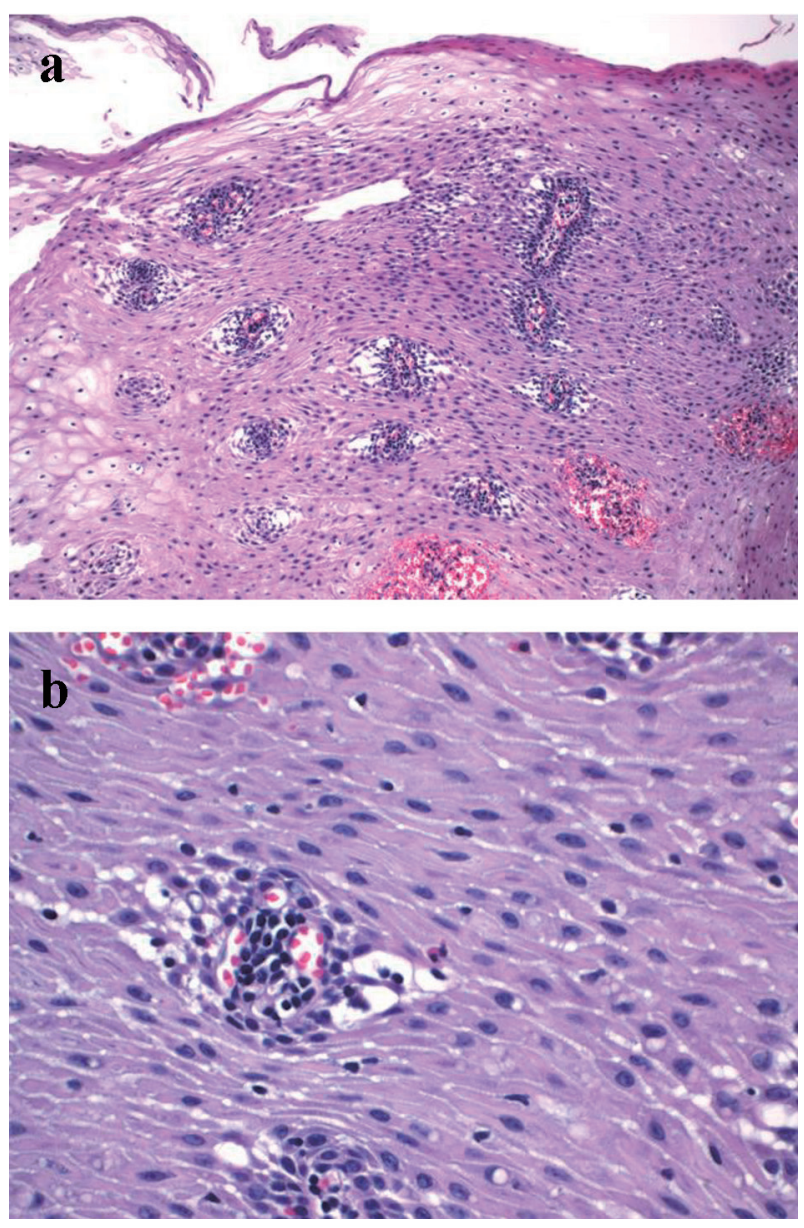

Figure 1. Mucosal changes in mid esophageal biopsy from one patient with achalasia who underwent peroral endoscopic myotomy. (a) Peripapillary edema (spongiosis) and ballooning are noticeable (H\&E stain, $\times 100$ ). (b) Intraepithelial lymphocytosis is present (H\&E stain, $\times 200$ ).

esophageal muscular biopsies [12]. However, this study did not report the status of ganglion cells in these muscular biopsies or duration of achalasia in these patients.

Focal and mild perivascular and/or intravascular margination of inflammatory cells were noted in $29.4 \%$ of the cases in this study. Similar findings were illustrated in $28.6 \%$ of muscular biopsies taken during POEM in a recently published study [13] and in $17 \%$ of muscular biopsies obtained during laparoscopic myotomy surgery [14]. In contrast to the high rate of eosinophilic inflammation in the muscularis externa reported by Jin et al [13], we only identified mild eosinophilic infiltration (up to 25 and 50 eosinophils per HPF) in $11.7 \%$ of the cases. The reasons for the discrepancy between the current and previous studies may include different stage of achalasia, specimen type, biopsy level (lower esophageal sphincter, distal esophagus proximately $5 \mathrm{~cm}$ above the EGJ, and mid esophagus (about $10-20 \mathrm{~cm}$ above the EGJ)) vs. mid esophagus only in the current study), and composition of different achalasia types/subsets. One study suggested the presence of two subsets of achalasia with one subset with T-cell-rich inflammation in the myenteric plexus and one subset with greater loss of nerve
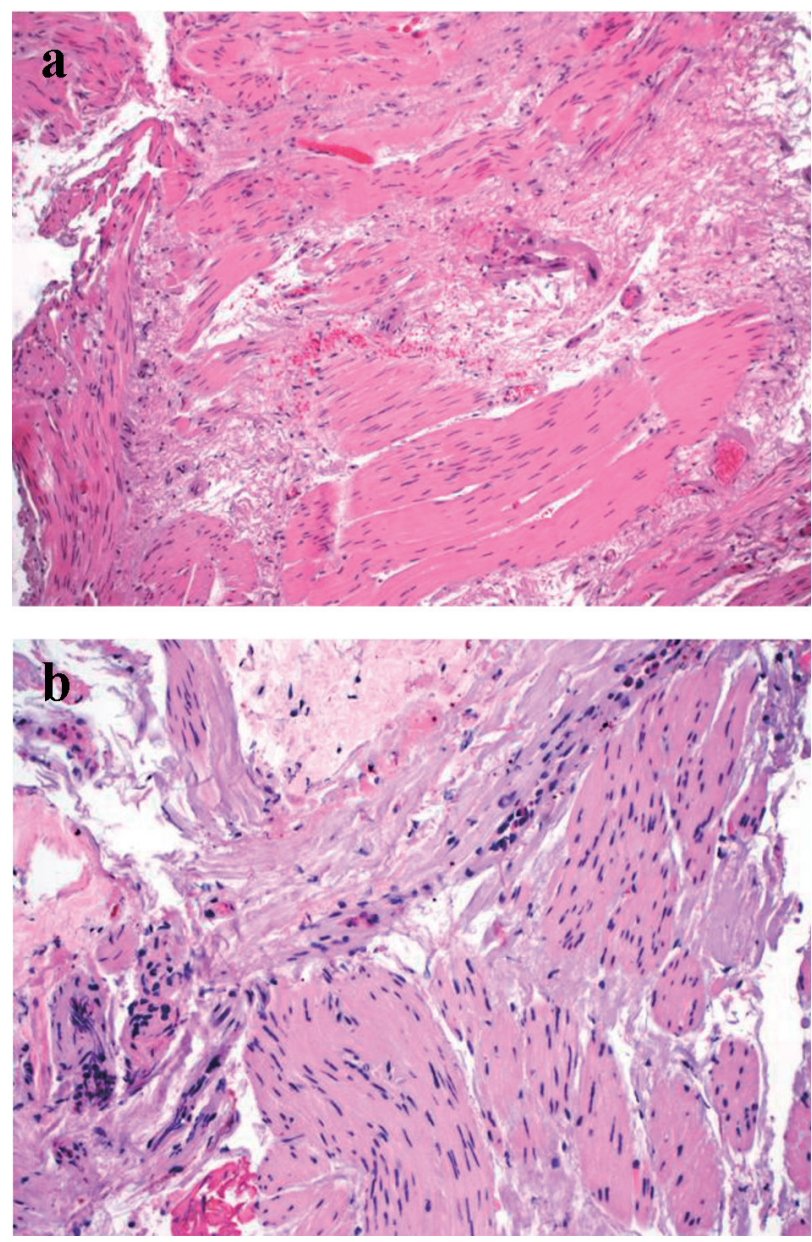

Figure 2. Finding in muscular biopsy in achalasia. (a) Interstitial fibrosis in the muscular biopsy (H\&E stain, $\times 100)$. (b) Interstitial fibrosis and elastosis and absence of ganglion cells in the muscular biopsy. The fibrosis/elastosis is accompanied by mild eosinophilic inflammation (H\&E stain, $\times$ 200).

fibers but without much inflammation [15]. The latter subset were comprised of patients with shorter duration of achalasia (6.4 years), suggesting a more aggressive disease [15]. The mean duration of achalasia in our patient population was 79 months, similar to the subset of achalasia with more aggressive disease [15], longer than the 14-month duration of disease in two studies showing more prominent inflammation in myotomy specimens $[2,11]$, shorter than the 10-year duration of disease who underwent esophagectomy [11]. One additional study of muscular biopsy obtained during laparoscopic myotomy surgery revealed different histopathologic patterns among achalasia subtypes with type I showing more prominent ganglion cell loss than type II [14]. The discrepancy may also be due to different patient population (Chinese vs. United States) and different definitions of muscularis propria/muscularis externa. Exact definitions of muscularis externa and muscularis propria were not given in the study by Jin et al [13]. Generally speaking, muscularis externa and muscularis propria refer to similar layer of esophagus, i.e., the muscle between the submucosa and the adventitia. It is not clear why the authors spe- 

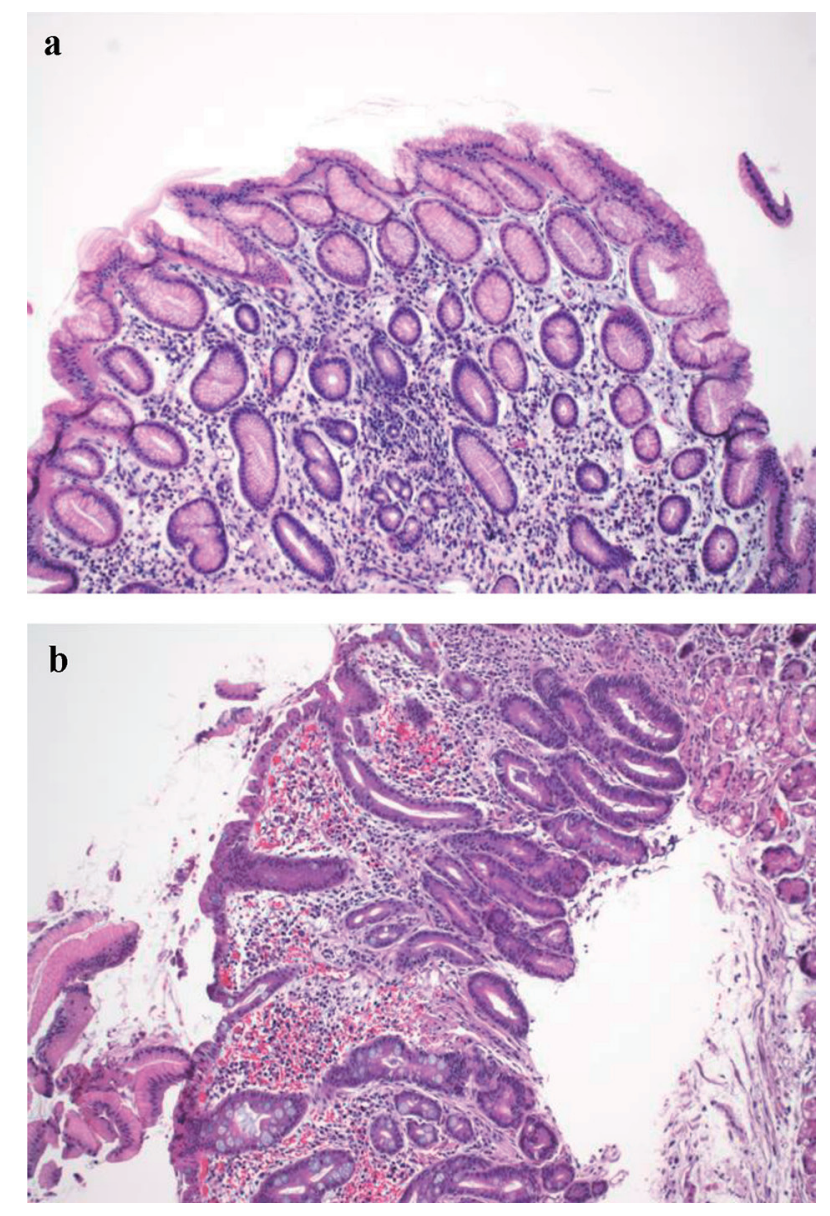

Figure 3. Histologic findings in the gastric cardia in achalasia. (a) Gastric cardia with mild chronic carditis (H\&E stain, $\times 100)$. (b) Moderate chronic carditis with intestinal metaplasia and focal low-grade dysplasia (H\&E stain, $\times 100)$.

cifically examined and reported eosinophilic infiltration in the muscularis propria and muscularis externa in that study [13].

In addition to the finding of ganglion cell loss in achalasia, the current study revealed muscular atrophy and fibrosis in $52.9 \%$ and $80 \%$ of cases. This is consistent with one previous study which reported a severe fibrosis of the smooth muscle layer in 10 of 17 cases and obvious myopathic changes of the smooth muscle cells in five of 17 cases [3].

It is well known that both eosinophilic and lymphocytic esophagitis, characterized by infiltration of eosinophils and lymphocytes in the mucosa, can manifest clinically with dysphagia. Whether eosinophilic/lymphocytic mural infiltration of the muscle layer of the esophagus is routinely present and plays a role in achalasia remains to be determined [4, 5]. Recent literature suggests an association of achalasia with eosinophilic esophagitis [4]. Mucosal changes at the time of POEM have not been studied. Our study aimed to evaluate the mucosal findings in the esophagus and gastric cardia immediately prior to POEM. The squamous mucosa biopsies showed a pattern of lymphocytic esophagitis, i.e., basal hyperplasia, papillary elongation, spongiosis, ballooning, and intraepithelial lymphocytes in $70.5 \%$ cases, resembling gastroesophageal reflux disease (GERD)-associated esophageal mucosa changes [8], and in line with a previous study of mucosal changes in the esophagectomy specimens for end-stage achalasia [3, 7]. This change may likely be due to stasis, although it resembles squamous changes in GERD. Lymphocytic inflammation in the muscular biopsy was only encountered in $5.8 \%$ of the cases in our study, which is much lower than the $70.5 \%$ of mucosal intraepithelial lymphocytosis rate, indicating a mucosa-accented lymphocytic inflammation in achalasia, likely secondary to stasis.

A few recent reports suggest that achalasia may be associated with eosinophilic esophagitis $[4,13]$. None of our cases showed mucosal eosinophilic inflammation to the level of eosinophilic esophagitis or diffuse eosinophilic inflammation in the muscular propria, suggesting that eosinophilic esophagitis is not likely the reason/etiology for/of majority of achalasia cases. However, the small size of our study does not rule out eosinophilic esophagitis as the etiology of achalasia in rare cases. Long-term clinical and manometric follow-up patients with eosinophilic esophagitis may provide a more definitive answer in the future.

Achalasia is a risk factor of squamous cell carcinoma [1, 16-19] and an increased frequency of p53 immunoreactivity has been reported in the esophageal mucosa in patients with late-stage achalasia [7]. Nonetheless, none of our patients showed squamous dysplasia, but this may have been precluded by the small sample size in this pilot study.

Gastric cardia was biopsied in 13 cases in the current study. No antrum or gastric body mucosa biopsies were obtained. Histologic findings included carditis (69.2\% of patients), $H$. pylori gastritis ( $7.6 \%$ of patients), and reactive gastropathy (15.4\% of patients). The prevalence of $H$. pylori gastritis was similar to the general population in the USA [20]. The etiology and mechanism of carditis in achalasia remains unclear. The peak acid output has been shown to be significantly lower in achalasia patients along with loss of Auerbach's plexus in the body of the stomach [21]. One case in our study $(7.6 \%)$ had low-grade dysplasia arising from intestinal metaplasia in the cardia with moderate chronic inactive carditis in the absence of H. pylori organisms on H\&E-stained section. No special stain or immunostain was performed for $H$. pylori in this case, so $H$. pylori gastritis-associated intestinal metaplasia-dysplasia cannot be completely ruled out. Achalasia secondary to malignancy in the cardia/esophagus is a well-known phenomenon; however, our current finding of low-grade dysplasia in the cardia in patients with achalasia suggests that "secondary" achalasia in cases with cardia/esophageal adenocarcinoma may be primary or idiopathic in some cases. Low-grade glandular dysplasia in this study was most likely of cardia origin rather than esophageal origin (i.e., Barrett's esophagus) as there was no endoscopic evidence of Barrett's esophagus. Interestingly, 61.1\% of patients were on PPIs at the time of POEM but only two patients had prior myotomy procedures. Our results suggest that biopsies of the stomach and esophageal mucosa at the time of POEM or before POEM should be performed to diagnose H. pylori infection and detect carditis, intestinal metaplasia, and dysplasia in these patients, albeit future studies are needed.

None of our patients had complications after cold biopsy and POEM. This is consistent with previous studies that the 
estimated risk of bleeding during or after biopsy is $0.002 \%$, extremely low [22]. Our results indicate that muscular biopsy is safe and may be used in patients who undergo POEM. Our results demonstrated that loss of ganglion cells was identified in most achalasia cases, suggesting that muscular biopsy obtained during endoscopy was adequate to study myenteric plexus and muscularis propria in achalasia. This muscular biopsy approach may be used in achalasia patients shortly after the onset of symptoms to obtain high-quality near full-thickness neuromuscular biopsies. Information on earlier changes in the myenteric plexus and muscularis propria in achalasia is essential for understanding the pathogenesis and may potentially yield effective medical non-invasive treatment strategies.

Our current study has several strengths and limitations. First, this is the first study to examine the esophageal mucosa, gastric cardia, and muscular biopsy in achalasia. Second, the muscular biopsy was done endoscopically at the time of POEM. Third, the histology was read by a gastrointestinal pathologist with an interest in esophageal diseases. One of the main limitations is the small size of the cohort which was partly due to the disruption of enrollment as a result of the COVID-19 pandemic. Another limitation was the lack of a normal control group for the muscular biopsy. However, biopsy of deep muscle in the esophagus in patients without an indication appears to be unethical. In addition, there are several previous studies examining normal esophageal muscle from the histologically normal esophageal segment from the esophagectomy performed for carcinoma [2, 13].

In summary, the current study demonstrated the feasibility and safety of deep muscle biopsy prior to POEM. Absence of ganglion cells was noted in the majority of achalasia patients undergoing POEM suggesting that our study patients were closer to end-stage disease, rather than early stage. Eosinophilic inflammation was only present in a minority of achalasia muscular biopsies and often of mild degree suggesting eosinophilic inflammation was not a driving force leading to achalasia in most cases. Achalasia-associated mucosal changes include a lymphocytic esophagitis pattern and chronic carditis which may lead to intestinal metaplasia and dysplasia.

\section{Acknowledgments}

None to declare.

\section{Financial Disclosure}

None to declare.

\section{Conflict of Interest}

None to declare.

\section{Informed Consent}

Informed consent has been obtained from the patients.

\section{Author Contributions}

XL and PD: study design, supervision, data analysis and manuscript drafting; EK, KW, and YBP: data collection and critical review of manuscript; DY: patient recruitment, data collection, and critical review of manuscript.

\section{Data Availability}

The authors declare that data supporting the findings of this study are available within the article.

\section{References}

1. Goldblum JR, Whyte RI, Orringer MB, Appelman HD. Achalasia. A morphologic study of 42 resected specimens. Am J Surg Pathol. 1994;18(4):327-337.

2. Goldblum JR, Rice TW, Richter JE. Histopathologic features in esophagomyotomy specimens from patients with achalasia. Gastroenterology. 1996;111(3):648-654.

3. Gockel I, Bohl JR, Doostkam S, Eckardt VF, Junginger T. Spectrum of histopathologic findings in patients with achalasia reflects different etiologies. J Gastroenterol Hepatol. 2006;21(4):727-733.

4. Mandaliya R, DiMarino AJ, Cohen S. Association of achalasia and eosinophilic esophagitis. Indian J Gastroenterol. 2013;32(1):54-57.

5. Lisovsky M, Westerhoff M, Zhang X. Lymphocytic esophagitis: a histologic pattern with emerging clinical ramifications. Ann N Y Acad Sci. 2016;1381(1):133-138.

6. Zaninotto G, Bennett C, Boeckxstaens G, Costantini M, Ferguson MK, Pandolfino JE, Patti MG, et al. The 2018 ISDE achalasia guidelines. Dis Esophagus. 2018;31(9):doy071.

7. Lehman MB, Clark SB, Ormsby AH, Rice TW, Richter JE, Goldblum JR. Squamous mucosal alterations in esophagectomy specimens from patients with end-stage achalasia. Am J Surg Pathol. 2001;25(11):1413-1418.

8. Dunbar KB, Agoston AT, Odze RD, Huo X, Pham TH, Cipher DJ, Castell DO, et al. Association of acute gastroesophageal reflux disease with esophageal histologic changes. JAMA. 2016;315(19):2104-2112.

9. Goldblum JR, Liu X. Role of histology and cytology in esophageal diseases. In: Richter JE, Castell DO, eds. The esophagus. Oxford: Wiley-Blackwell; 2012.

10. Rouphael C, Gordon IO, Thota PN. Lymphocytic esophagitis: Still an enigma a decade later. World J Gastroenterol. 2017;23(6):949-956.

11. Clark SB, Rice TW, Tubbs RR, Richter JE, Goldblum JR. The nature of the myenteric infiltrate in achalasia: an immunohistochemical analysis. Am J Surg Pathol. 2000;24(8):1153-1158.

12. Reddy CA, Law R, Appelman HD, Chang AC, Korsnes $\mathrm{S}$, Chen JW. Per-oral endoscopic myotomy biopsies of achalasia patients reveal Schwann cell depletion in the muscularis propria. Clin Gastroenterol Hepatol. 
2021;19(6):1294-1295.

13. Jin H, Wang B, Zhang LL, Zhao W. Activated Eosinophils are Present in Esophageal Muscle in Patients with Achalasia of the Esophagus. Med Sci Monit. 2018;24:23772383.

14. Sodikoff JB, Lo AA, Shetuni BB, Kahrilas PJ, Yang GY, Pandolfino JE. Histopathologic patterns among achalasia subtypes. Neurogastroenterol Motil. 2016;28(1):139-145.

15. Kilic A, Krasinskas AM, Owens SR, Luketich JD, Landreneau RJ, Schuchert MJ. Variations in inflammation and nerve fiber loss reflect different subsets of achalasia patients. J Surg Res. 2007;143(1):177-182.

16. Aggestrup S, Holm JC, Sorensen HR. Does achalasia predispose to cancer of the esophagus? Chest. 1992;102(4):1013-1016.

17. Meijssen MA, Tilanus HW, van Blankenstein M, Hop WC, Ong GL. Achalasia complicated by oesophageal squamous cell carcinoma: a prospective study in 195 patients. Gut. 1992;33(2):155-158.

18. Peracchia A, Segalin A, Bardini R, Ruol A, Bonavina L,
Baessato M. Esophageal carcinoma and achalasia: prevalence, incidence and results of treatment. Hepatogastroenterology. 1991;38(6):514-516.

19. Streitz JM, Jr., Ellis FH, Jr., Gibb SP, Heatley GM. Achalasia and squamous cell carcinoma of the esophagus: analysis of 241 patients. Ann Thorac Surg. 1995;59(6):16041609.

20. Brown LM. Helicobacter pylori: epidemiology and routes of transmission. Epidemiol Rev. 2000;22(2):283-297.

21. Csendes A, Smok G, Braghetto I, Gonzalez P, Henriquez A, Csendes P, Pizurno D. Histological studies of Auerbach's plexuses of the oesophagus, stomach, jejunum, and colon in patients with achalasia of the oesophagus: correlation with gastric acid secretion, presence of parietal cells and gastric emptying of solids. Gut. 1992;33(2):150154.

22. Alneaimi K, Abdelmoula A, Vincent M, Savale C, Baye B, Lesur G. Seven cases of upper gastrointestinal bleeding after cold biopsy. Endosc Int Open. 2016;4(5):E583584. 